Document downloaded from:

http://hdl.handle.net/10251/46797

This paper must be cited as:

Cueto Belchí, AD.; Rothpfeffer, N.; Pelegrí Sebastiá, J.; Chilo, J.; García Rodríguez, D.; Sogorb Devesa, TC. (2013). Sensor characterization for multisensor odor-discrimination system. Sensors and Actuators A: Physical. 191:68-72. doi:10.1016/j.sna.2012.11.039.

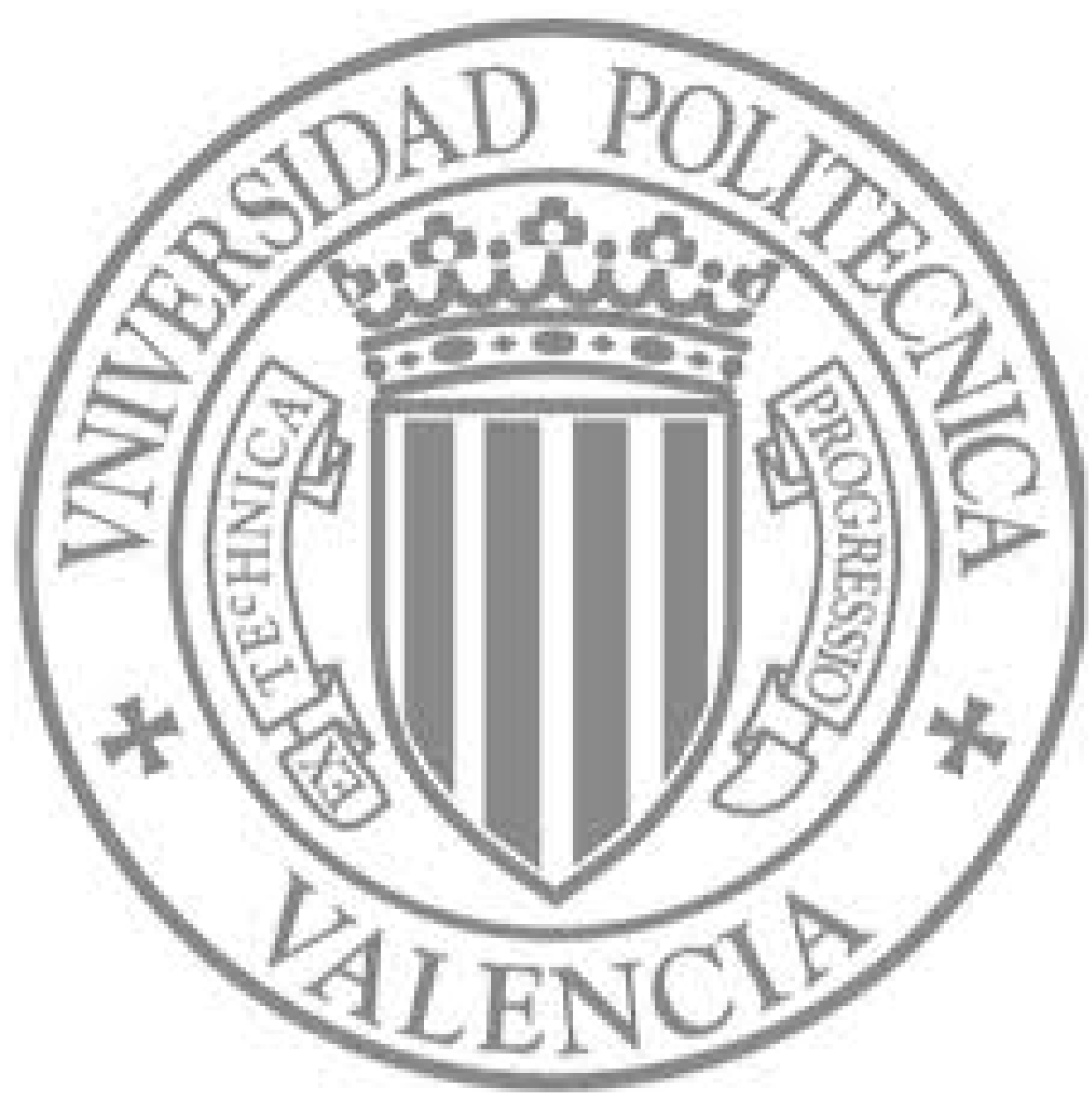

The final publication is available at

http://dx.doi.org/10.1016/j.sna.2012.11.039

Copyright Elsevier 


\section{Sensor Characterization for Multisensor Odor-Discrimination System}

A. del Cueto Belchi ${ }^{1}$, N. Rothpfeffer ${ }^{2}$, J. Pelegrí-Sebastia ${ }^{1}$, J. Chilo ${ }^{2}$, D. Garcia Rodriguez ${ }^{1}$ and T. Sogorb ${ }^{1}$

${ }^{1}$ Department of Electronic Engineering, Polytechnic University of Valencia

C/ Paranimf, 1, 46730 Grau de Gandia (Valencia), Spain,

${ }^{2}$ Department of Electronics and Physics, University of Gävle SE-80176, Sweden

Email: jpelegri@eln.upv.es (corresponding author) 
Abstract -In recent years, with the advent of new and cheaper sensors, the use of olfactory systems in homes, industries, and hospitals has a new start. Multisensor systems can improve the ability to distinguish between complex mixtures of volatile substances. To develop multisensor systems that are accurate and reliable, it is important to take into account the anomalies that may arise because of electronic instabilities, types of sensors, and air flow. In this approach, 32 metal oxide semiconductor sensors of 7 different types and operating at different temperatures have been used to develop a multisensor olfactory system. Each type of sensor has been characterized to select the most suitable temperature combinations. In addition, a prechamber has been designed to ensure a good air flow from the sample to the sensing area. The multisensor system has been tested with good results to perform multidimensional information detection of two fruits, based on obtaining sensor matrix data, extracting three features parameters from each sensor curve and using these parameters as the input to a pattern recognition system.

$\underline{\text { Keywords }}$ - multisensor, sensor characterization, prechamber, feature extraction, classification

\section{INTRODUCTION}

A very intensive research about olfactory systems is going on with the objective to find new alternative methods for volatile organic compound classification in different areas. Now, researchers can obtain large and heterogeneous signal datasets generated in real time. The data are then processed with complex algorithms to extract information that is useful for applications such as quality and taste determination, leakage detection, food and medical diagnostics. However, it is precisely in medical applications that olfactory systems are very interesting, and several research projects are under way. One reason for this is the several dog projects that run around the world for medical diagnosis [1-3]. If a dog can be used for these medical applications, why not use an electronic olfactory system that offers greater advantages?

Here, we briefly review the progress in the field of artificial olfaction for medical applications. In [4], the authors present a networked electronic nose, which can detect and recognize human odors from the armpit region. Studies of characterization of breath from smokers and nonsmokers using electronic nose were reported in [5] with the objective of proving the influence of smoking 
on breath analyses. Moczko et al. present in [6] an optical diagnostic assay consisting of a mixture of environmental-sensitive fluorescent dyes combined with multivariate data analysis for quantitative and qualitative examination of biological and clinical samples using electronic nose and electronic tongue systems. Detection and diagnosis of lung cancer based on an electronic nose instrument that includes a gas extraction and capillary column to concentrate, desorb, and separate volatile organic compounds (VOCs) in patients' breath is found in a paper by Wang et al. [7]. References [8-9] aim to determine human blood glucose levels through analyzing the acetone present in exhaled breath as a noninvasive method with the help of an electronic nose system. Garcia-Cortes et al. propose in [10] a method to detect the presence of cortisol and adrenaline in body's sweat by electronic noses with resistive sensors as a noninvasive method. Finally, studies in [11] have shown the potential for early detection of microbial contaminants in urine samples using electronic nose technology.

A preliminary study of our work was presented in [12] where we use 32 gas sensors from Figaro Engineering Inc. to develop an olfactory system that can be used to classify a wide variety of volatile organic compounds. Some related applications that have been developed based on Figaro gas sensors are breath alcohol checkers, automatic cooking controls in microwave ovens, and air quality/ventilation control systems for home and automobiles. Figaro gas sensors are solid-state sensors composed of a sintered metal oxide that detects gases through an increase in electrical conductivity when reducing gases are adsorbed on the sensor's surface.

A simple electrical circuit shown in Fig. 1 can convert the change in conductivity to an output signal that corresponds to the gas concentration. The sensor requires two voltage inputs: heater voltage $\left(\mathrm{V}_{\text {Heater }}\right)$ and circuit voltage $\left(\mathrm{V}_{\text {sensor }}\right)$. The heater voltage $\left(\mathrm{V}_{\text {Heater }}\right)$ is applied to the integrated heater to maintain the sensing element at a specific temperature, which is optimal for sensing. 
Circuit voltage $\left(\mathrm{V}_{\text {sensor }}\right)$ is applied to allow measurement of voltage $\left(\mathrm{V}_{\text {out }}\right)$ across a load resistor $\left(R_{L}\right)$, which is connected in series with the sensor [13-14].

The ability to have different sensitivity properties by selecting the most appropriate combinations of the sensor temperature $\left(\mathrm{V}_{\text {Heater }}\right)$ and the possibility that Figaro Engineering Inc. provides various types of sensors enables the development of a multisensor olfactory system with 32, 64, 128 , or more sensors.

Thereafter, the output signals from the sensors are digitized and stored for each sample. In this work three features parameters are extracted from each sensor curve: transient slope, saturation slope and maximum slope. These parameters are used as the input to a pattern recognition system to classify the substance under test.

\section{OLFACTORY SYSTEM}

\section{A. Sensor Characterization}

The basic assumption behind our olfactory system is that normal variations in the MOS sensors from Figaro Engineering Inc. combined with the selected operating temperature lead to a wide range of different responses toward volatile organic compounds. We have used seven commercial MOS that are sensitive to various types of gases: TGS2610-C00, TGS2610-D00, TGS2611-C00, TGS2600-B00, TGS2620-C00, TGS2602-B00, and TGS2442-B02. Because of the high sensitivity of these sensors, they are used in residential gas alarms, gas leak checkers, indoor air quality controls, and residential CO detectors. Subsequently, these sensors can be beneficially used in more complex applications, such as in medicine to detect various diseases, particularly different types of cancers. 
Figaro sensors require a small amount of power to operate the sensor element at elevated temperatures between $200^{\circ} \mathrm{C}$ to $400^{\circ} \mathrm{C}$. By varying the operating temperature, changing $\mathrm{V}_{\text {Heater, }}$, we can alter the sensor's response to various combustible gases. The seven types of sensors used in this work were investigated with the measurement setup illustrated in Fig. 2 to determine their $\mathrm{V}_{\text {Heater }}$ range.

The sensor characterization system is based on a virtual instrumentation system, where two instruments are connected to a computer via GPIB bus, and the LabVIEW is used to handle them. A power source (FA-851) supplies two drive currents, one for the sensor (Rs) and the other for the heater (Rh). Then, a digital multimeter (HP 33120A) is used to measure the output voltage of the sensor on the load resistor $\left(\mathrm{R}_{\mathrm{L}}\right)$. Table I lists the range results in the form of current instead of voltage for some sensors.

\section{B. System Design}

The complete system is shown in Fig. 3 consisting of two parts: the electrical part and the part of the preprocessing of the samples, the prechamber. The electrical design of the system consists of eight identical electronic boards with four sensors in each of them. Each board includes a voltage regulator (LP38692MP-ADJ), which supplies the heater ( $\left.\mathrm{V}_{\text {Heater }}\right)$ for each of the four sensors. This regulator is controlled by the user interface and allows us to vary the supply voltage during the measurement process. The output voltage $\left(\mathrm{V}_{\text {out }}\right)$ of each sensor is fed into an amplifier (OP074) in a voltage follower configuration.

For data acquisition, we use an ADC from the National Instruments (NI USB-6218, 16 Bits, 250 kS/s, Isolated M. Series MIO DAQ, Bus-Powered). The NI USB-6218 has 32 analog inputs that we use for data collection from the 32 sensors and only two analog outputs that are used to 
adjust the output voltage of the regulator and to control the electrovalve. In addition, it has 32 digital I/O lines that can be used to control some functions of the system by software.

The first part of the prechamber has a clean air pump. The air flow is adjusted manually with a small valve. This air is split into two streams, one that goes through the sample chamber and thereafter to the electrovalve and the other that goes directly to the electrovalve. The electrovalve is controlled automatically from the interface. The process is as follows: clean air passes through the system to clean it during a predetermined time and then passes air flow through the sample chamber during another fixed time.

The setup of the parameter for the measurements is controlled by a dedicated graphical user interface (GUI) implemented using LabVIEW on a personal computer [15]. This highly GUI allows to configure the hardware with full control and flexibility.

From the GUI, we can configure individually each sensor. Figure 4 shows the configuration panel in different colors corresponding to the eight electrical boards.

\section{DATA ANALYSIS}

For classification, the analysis process involves presenting the multisensor measurements to the input of a classification machine for a given slice of time. However, the raw data can be quite large and/or noisy to be effective, so a preprocessing step is needed to reduce the raw data to a smaller input data set that highlights particular features in the signals. In our case, data preprocessing is done using the software functions from LabVIEW. For example, we have designed an IIR low-pass digital filter of second order with variable cutoff frequency to eliminate any possible ripple. 
The measurement steps used in our system are presented in Fig. 5. The first step in the olfactory analysis is to present a reference gas (clean air) to the sensor to obtain a reference line. Then the sensor is exposed to an odoriferous substance, causing changes in its output until the sensor reach a steady state. Finally, the odorant is flushed out of the sensor using the reference gas, and the sensor returns to its original state. From the software, we can set the open, acquisition, and close times depending on the substance to be analyzed.

The classification features used in our system are presented in Fig. 6 and are late saturation, saturation slope, early saturation, transient slope, and time to threshold [16-17].

The system has been tested with a number of substances working well. The analysis presented here is on the response of two fruits: pear and apple. Ten samples of every one. The output of the sensors consists of 32 independent analog voltages, each varying with time and odor. The voltage is sampled at 100 samples/second, and the raw data file is compacted to a data matrix consisting of 32 columns by 2000 rows.

As in previous works [18], the sampled signals are reduced to three numbers for each sensor: transient slope, saturation slope, and late saturation - three features that are believed to describe the origin of the signals well.

Pattern recognition algorithms are necessary to extract useful information from sensor outputs. One of the most common pattern recognition techniques used is principal component analysis (PCA) [19-20]. PCA is a linear data reduction technique, which reduces multivariate data into two or three dimensions. This technique extracts features by projecting the high-dimensional data set into a dimensionally reduced space formed by the uncorrelated and orthogonal eigenvectors of the covariance matrix calculated from the sensor responses, called principal components. The magnitude of the single eigenvector or percentage of information is expressed by the eigenvalue, which gives a measure of the variance related to that principal component [21]. 
This work uses PCA to visualize the similarities and differences between the various measurements in the dataset, using the first feature, transient slope. Fig. 7 shows the result plot of data in the PC1-PC2-PC3 plane of pear and apple. It is shown that the measurements are divided into two different groups.

The primary data are the 32 signals from the sensors (different sensors operating at different temperatures each) which are digitized and stored for each sample. Three features parameters are extracted from each sensor curve (transient slope (TS), saturation slope (SS) and maximum slope (MS) when the sample is closed), making a total of 96 parameters for each sample. Those parameters are used as the input to the pattern recognition systems: WEKA [22]. This is an open source data mining toolbox (written in Java) developed by Ian Witten's group at the University of Waikato. It provides tools for all the tasks usually performed in data mining, including numerous algorithms for pre-processing, classification, regression and clustering. Here we utilized 10 different classification algorithms in WEKA. We used their default parameters unless otherwise stated.

Here are the classification algorithms used in our work with their default parameters unless otherwise stated:

- BayesNet creates a Bayesian Network with implementation of the standard naïve Bayes algorithm, where normal distribution is for numerical features.

- $\quad$ RBFNetwork is an implementation of radial basis functions.

- SimpleLogistic constructs linear logistic regression models.

- SMO is a sequential minimum optimization algorithm for building support vector machine. 
- IB1 is a nearest-neighbor algorithm that classifies an instance according to the nearest neighbor identified by the Euclidean distance.

- The Kstar algorithm uses entropic distance measure, based on the probability of transforming one instance into another by randomly choosing between all possible transformations, and turns out to be better than Euclidean distance for classification.

- VFI finds intervals for each feature and attributes each class according to the number of instances with the class in the training set for the specific interval. Voting is used to select the final class for an instance.

- ADTree algorithms create trees as models.

- NNge is a nearest-neighbor algorithm that learns rules based on the hyper rectangles that it divides the instance space.

- PART constructs rules based on partial decision treesOur inexhaustible power source supplies.

Table II gives the results for 20 runs (10 pears and 10 apples) from our experiment.

\section{CONCLUSION}

This work has used 32 gas sensors (seven different types of sensors) from Figaro Engineering Inc. to develop an olfactory system that can be used to classify a wide variety of volatile organic compounds. A wide range of responses can be obtained by selecting the most appropriate combinations of the sensor working temperature. To know the most suitable temperature combinations, we have characterized each sensor. 
The olfactory system has been verified with tests of two fruits, pear and apple, and classification algorithms such as PCA and others found in WEKA with good results.

\section{REFERENCES}

[1] R.T. Gordon, C.B. Schatz, L.J. Myers, M. Kosty, C. Gonczy, J. Kroener, M. Tran, P. Kurtzhals, S. Heath, J.A. Koziol, N. Arthur, M. Gabriel, J. Hemping, G. Hemping, S. Nesbitt, L. Tucker-Clark and J. Zaayer, “The use of canines in the detection of human cancers”, Journal of Alternative and Complementary Medicine, Vol. 14, Issue 1, pp. 61-67, Jan. 2008.

[2] J.N. Cornu, G. Cancel-Tassin, V. Ondet, C. Girardet and O. Cussenot, “Olfactory detection of prostate cancer by dogs sniffing urine: a step forward in early diagnosis”, European Urology, Vol. 59, No. 2, pp. 197-201, Feb. 2011.

[3] Y. Hanai, K. Shimono, H. Oka, Y. Baba, K. Yamazaki and G.K. Beauchamp, “Analysis of volatile organic compounds released from human lung cancer cells and from the urine of tumor-bearing mice”, Cancer Cell International, Vol. 12, No. 7, pp. 1-13, Jan. 2012.

[4] C. Wongchoosuk, M. Lutz, T. Puntheeranurak, T. Youngrod, H. Phetmung, and T. Kerdcharoen, ”Identification of people from armpit odor region using networked electronic nose”, IEEE Defense Science Research Conference and Expo (DSR), pp. 1-4, Aug. 2011.

[5] K. Witt, S. Reulecke and A. Voss, "Discrimination and characterization of breath from smokers and nonsmokers via electronic nose and GC/MS analysis”, IEEE Conference Engineering in Medicine and Biology Society EMBC, pp. 3664-3667, Sept. 2011.

[6] E. Moczko, M. Cauchi, C. Turner, I. Meglinski, and S. Piletsky, ”Optical assay for biotechnology and clinical diagnosis”, IEEE Transactions on Biomedical Engineering, Vol. 58, Issue 8, pp. 2154-2160, 2011.

[7] P. Wang, X. Chen, F. Xu, D. Lu, W. Cai, K. Ying, Y. Wang and Y. Hu, ”Development of electronic nose for diagnosis of lung cancer at early stage”, IEEE Conference Information Technology and Applications in Biomedicine, pp. 588-591, May 2008. 
[8] H.M.Saraoglu and M. Kocan, "Determination of blood glucose level-based breath analysis by a quartz crystal microbalance sensor array”, IEEE Sensors Journal, Vol. 10, Issue 1, pp. 104-109, 2010.

[9] H.M Saraoğlu and M. Koçan, "Determination of diabetic blood glucose value from breath odor using QCM sensor based Electronic Nose”, IEEE Conference Biomedical Engineering Meeting (BIYOMUT), pp. 1-4, April 2010.

[10] A. Garcia-Cortes, J. Marti, I. Sayago, J.P. Santos, J. Gutierrez and M.C. Horrillo, "Detection of stress through sweat analysis with an electronic nose”, IEEE Conference Electron Devices, pp. 338-341, Feb. 2009.

[11] V.S. Kodogiannis, J.N. Lygouras, A. Tarczynski and H.S. Chowdrey, “Artificial odor discrimination system using electronic nose and neural networks for the identification of urinary tract infection”, IEEE Transactions on Information Technology in Biomedicine, Vol. 12 , Issue 6, pp. 707-713, 2008.

[12] A. Del Cueto Belchi, D. Carcia Rodriguez, N. Rothfeffer, J. Pelegri Sebastiá and J. Chilo, “Multi-sensor olfactory system: using temperature modulation”, IEEE International Instrumentation and Measurement Technology Conference, pp. 1139-1141, May 2012.

[13]J.G. Webster, "The measurement, instrumentation and sensors" handbook. CRC Pres Inc, ISBN: 9780849383472, 1999.

[14]R. Pallás-Areny and J.G. Webster, "Sensors and Signal Conditioning”, Ed. John Wiley and Sons, New York, ISBN: 978-0471332329, 1991.

[15]R. Lajara and J. Pelegri, “LabVIEW Entorno gráfico de Programación”, Ed. Marcombo, Barcelona, ISBN: 9788426716965, 2011.

[16] J. Waldemark, T. Roppel, M. Padgett, D. Wilson and Th. Lindblad, "Neural network and PCA for determining region of interest in sensory data pre-processing”, Virtual Intelligence/Dynamic Neural Network Workshop, vol. 3728, pp. 396-405, 1998.

[17] M. Kermit, Å. J. Eide, Th. Lindblad and K. Agehed, “Intelligent machine olfaction”, IASTED, Tokio, Japan, pp. 25-27, 2002.

[18] G. Horvath, J. Chilo and Th. Lindblad, "Different volatile signals emitted by human ovarian carcinoma and healthy tissue”, Future Oncology, vol. 6, Nr 6, pp. 1043-1049, June 2010.

[19]F.Sarry and M. Lumbreras, "Gas discrimination in an air conditioned system”, IEEE Trans. Instrument. Measurement, vol. 49, Nr. 4, pp. 809-812, 2010. 
[20] J.W. Gardner, "Detection of vapours and odours from a multisensor array using pattern recognition: Principal component and cluster analysis”, Sensors Actuators, vol. 4, pp.109-115, 1991.

[21]N. El Barbri, E. Llobet, N. El Bari, X. Correig and B. Bouchikhi, ”Electronic nose based on metal oxide semiconductor sensors as an alternative technique for the spoilage classification of red meat”, Sensors, vol. 8, pp. 142-156, 2008.

[22] I.H. Witten and E. Frank, “Data mining: Practical Machine Learning Tools and Techniques”, $2^{\text {nd }}$ Edition, Morgan Kaufmann Publishers, San Mateo, CA., 2005. 


\section{Figure and Table Captions}

Figure. 1. Basic measuring circuit.

Figure 2. Sensor characterization setup.

Figure 3. The complete system: electrical design of the system and the prechamber.

Figure 4. Sensor configuration panel.

Figure 5. Measurement steps.

Figure 6. Classification features.

Figure 7. PCA using Transient Slope.

TABLE I.RANGE OF SENSOR TEMPERATURES

TABLE II.CLASSIFICATION RESULTS WITH WEKA 

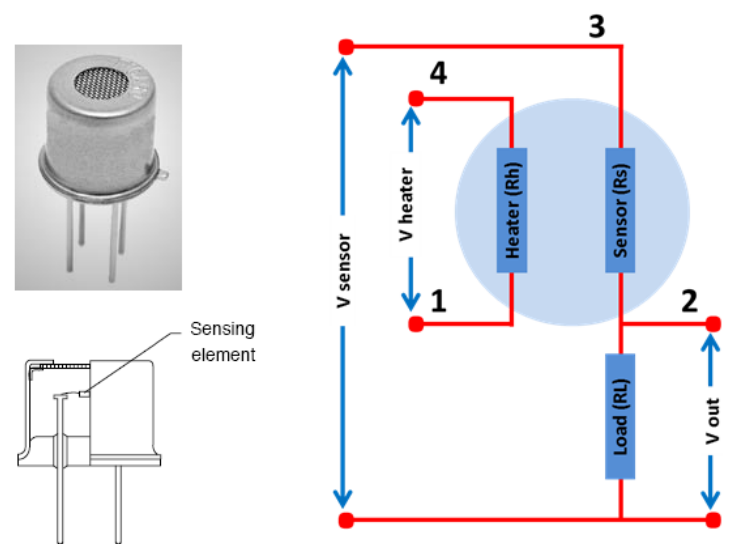

Figure 1. Basic measuring circuit 


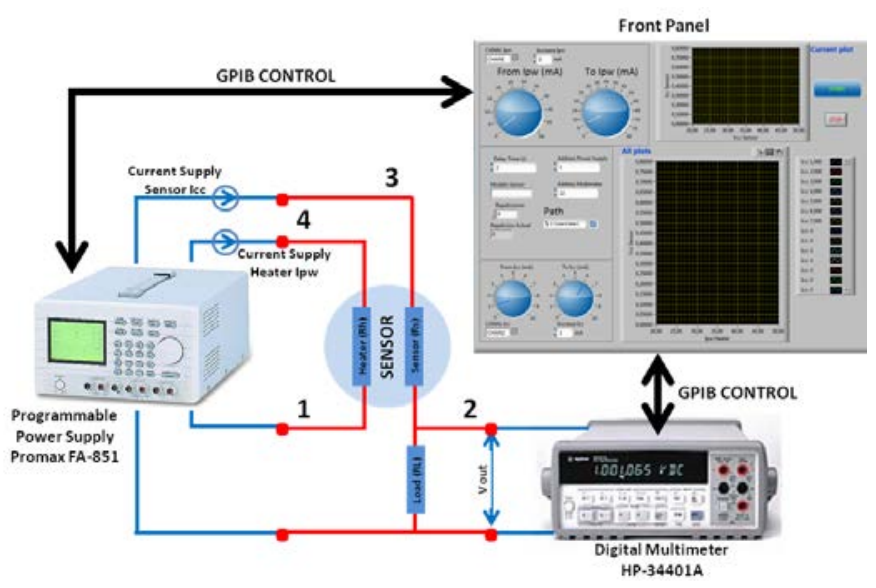

Figure 2. Sensor characterization setup 


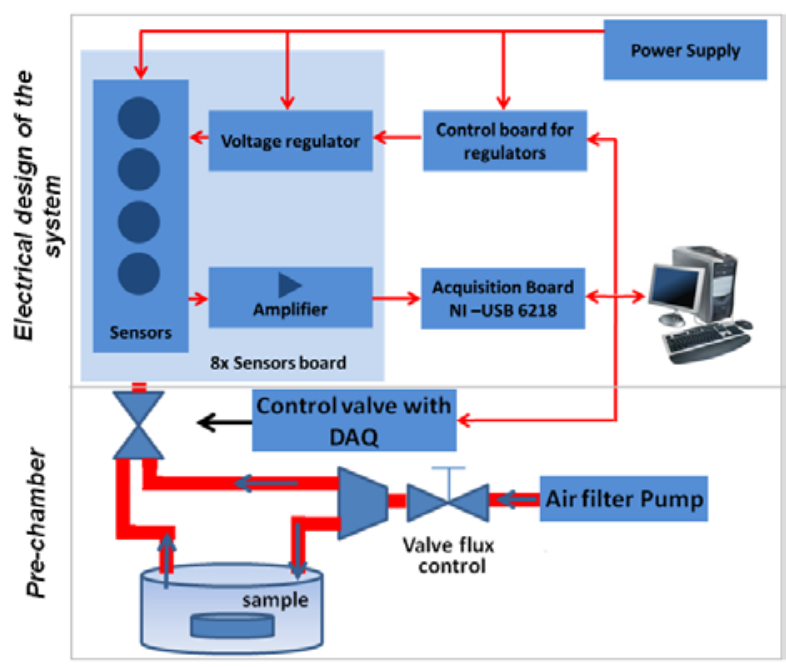

Figure 3. The complete system: electrical design of the system and the prechamber 


\begin{tabular}{|c|c|c|c|}
\hline \multicolumn{2}{|c|}{ minimum value maximum value } & \multicolumn{2}{|c|}{ CHANNEL CONFIG } \\
\hline Channel 1 TGS 2620 & Channel 9 TGS 2620 & Channel 17 TGS 2620 & Channel 25 TGS 2620 \\
\hline$\nabla$ Jover/aio $\square$ & $\nabla$ I/o Dev2/ai8 $=$ & $\nabla$ I/o Dev2/ail6 - & $\nabla$ I/o Dev2/aiz4 $=$ \\
\hline Channel 2 TGS 2620 & Channel 10 TGS 2620 & Channel 18 TGS 2620 & Channel 26 TGS 2620 \\
\hline V I/0 Dev2/ail च & $\nabla /$ Dev2/ai9 & $\nabla \longdiv { \% }$ Dev2/ai17 & $\nabla$ I/0 Dev2/ai25 \\
\hline Channel 3 TGS 2620 & Channel 11 TGS 2620 & Channel 19 TGS 2620 & Channel 27 TGS 2620 \\
\hline$\nabla \mathrm{I} / \mathrm{Dev} 2 / \mathrm{ai} 2 \quad-$ & $\nabla \frac{\mathrm{T} / \mathrm{Dev} 2 / \mathrm{ai} 2 \quad \square}{\square}$ & $\nabla \longdiv { I _ { 0 } D e v 2 / a i 1 8 }$ & $\nabla$ I Dev2/ai26 $=$ \\
\hline Channel 4 TGS 2620 & Channel 12 TGS 2620 & Channel 20 TGS 2620 & Channel 28 TGS 2620 \\
\hline$\nabla \mathrm{I} / \mathrm{Dev} 2 / \mathrm{ai3} \quad \nabla$ & $\nabla \longdiv { I _ { 0 } \text { Dev2/ail1 } }$ & $\nabla \longdiv { 7 }$ Dev2/ai19 & $\nabla \frac{1}{10}$ Dev2/ai27 \\
\hline Channel 5 TGS 2610 & Channel13 TGS 2610 & Channel 21 TGS 2610 & Channel 29 TGS 2610 \\
\hline$\nabla \sqrt[T]{\mathrm{I} \text { Dev2/ai4 - }}$ & ( I/6 Dev2/ail2 - & $\nabla \longdiv { I _ { 0 } \operatorname { D e v } 2 / \mathrm { aiz } = }$ & $\nabla \longdiv { I _ { 0 } \text { Dev2/aiz8 } }$ \\
\hline Channel 6 TGS 2600 & Channel 14 TGS 2600 & Channel 22 TGS 2600 & Channel 30 TGS 2600 \\
\hline$\nabla$ I\%Dev2/ai5 - & ( I/6 Dev2/ail3 $=$ & 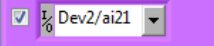 & $\nabla$ I/0 Dev2/aiz29 $=$ \\
\hline Channel 7 TGS 2620 & Channel 15 TGS 2620 & Channel 23 TGS 2620 & Channel 31 TGS 2620 \\
\hline 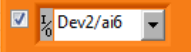 & VI/0 Dev2/ai14 - & $\nabla \longdiv { I _ { 0 } D e v 2 / a i 2 2 - V }$ & $\nabla \longdiv { I _ { 0 } \text { Dev2/ai30 } }$ \\
\hline Channel 8 TGS 2611 & Channel 16 TGS 2611 & Channel 24 TGS 2611 & Channel 32 TGS 2611 \\
\hline$\nabla \frac{I / 0 \text { Dev2/ai7 }}{-}$ & I/o Dev2/ail5 - & $\nabla \longdiv { I _ { 0 } \operatorname { D e v } 2 / \mathrm { ai } 2 3 - }$ & 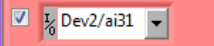 \\
\hline
\end{tabular}

Figure 4. Sensor configuration panel 


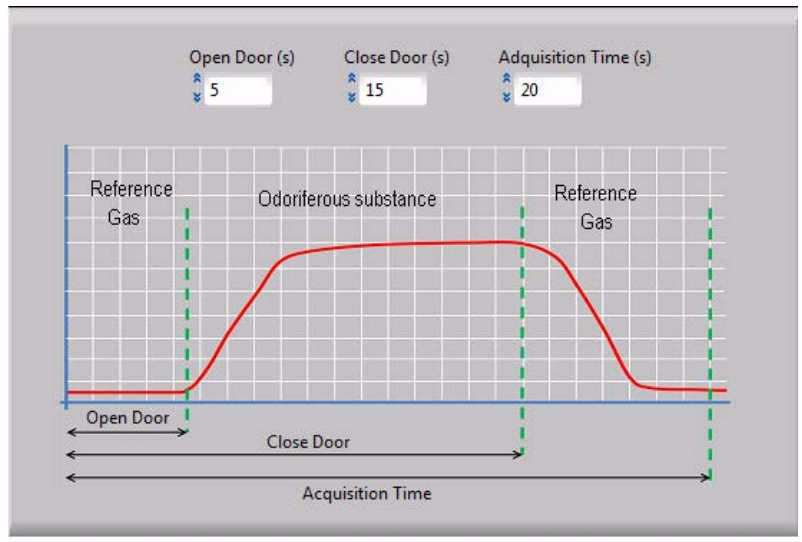

Figure 5. Measurement steps 


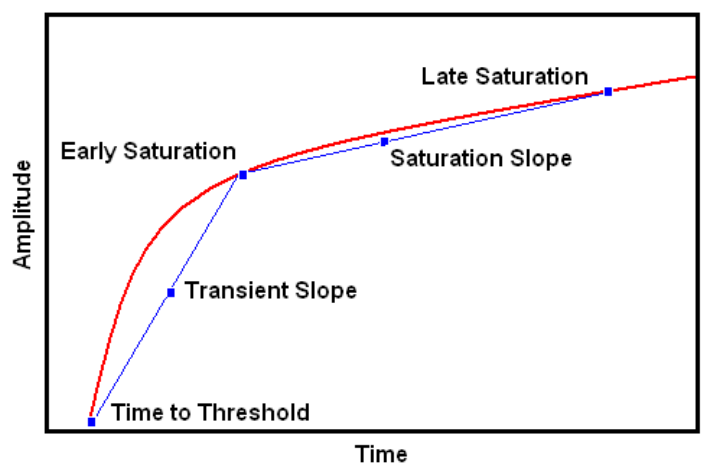

Figure 6. Classification features 


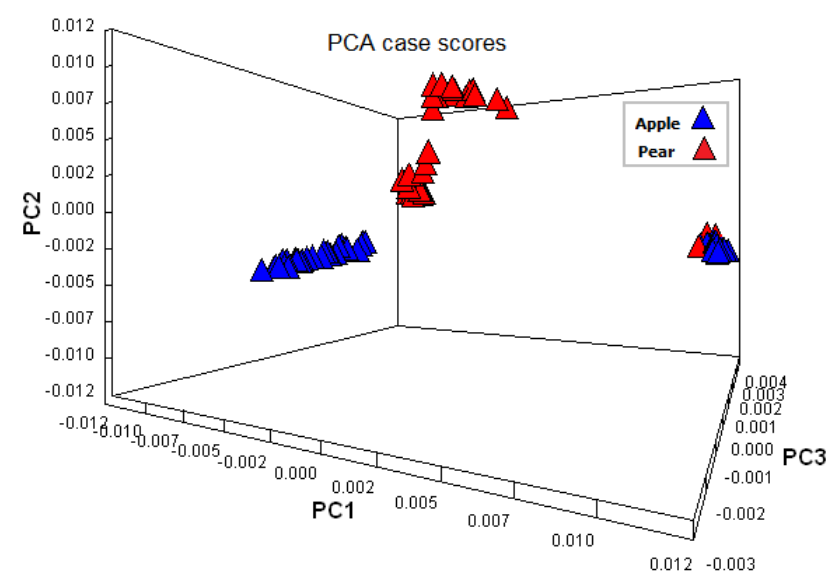

Figure 7. PCA using Transient Slope 
TABLE I. RANGE OF SENSOR TEMPERATURES

\begin{tabular}{|l|l|l|}
\hline Sensor & $\begin{array}{l}\text { Isensor } \\
\text { (mA) }\end{array}$ & $\begin{array}{l}\text { IHeater } \\
\text { (mA) }\end{array}$ \\
\hline TGS2600 & $2.5-6.0$ & $30-38$ \\
TGS2602 & $3.0-6.0$ & $35-45$ \\
TGS2610-C00 & $2.5-6.0$ & $30-45$ \\
TGS2610-D00 & $2.5-6.0$ & $30-45$ \\
TGS2611 & $2.5-6.0$ & $30-38$ \\
TGS2620 & $2.0-6.0$ & $25-35$ \\
TGS2442 & NC* & NC* \\
\hline
\end{tabular}

* Not Characterized due it uses different circuit diagram 
TABLE II. CLASSIFICATION RESULTS WITH WEKA 40 SAMPLES

\begin{tabular}{|l|l|l|l|}
\hline $\begin{array}{l}\text { Classification Al- } \\
\text { gorithm }\end{array}$ & Pear & Apple & $\begin{array}{l}\text { Total (\%) } \\
\text { Correctly } \\
\text { Classified }\end{array}$ \\
\hline Bayes Network & $37 / 39$ & $38 / 40$ & 94 \\
RBF Network & $38 / 39$ & $36 / 40$ & 93 \\
SimpleLogistic & $39 / 39$ & $38 / 40$ & 97 \\
SMO & $39 / 39$ & $39 / 40$ & 99 \\
IB1 & $39 / 39$ & $40 / 40$ & 100 \\
KStar & $39 / 39$ & $38 / 40$ & 97 \\
VFI & $34 / 39$ & $36 / 40$ & 89 \\
ADTree & $39 / 39$ & $36 / 40$ & 95 \\
NNge & $39 / 39$ & $38 / 40$ & 97 \\
PART & $38 / 39$ & $36 / 40$ & 94 \\
\hline
\end{tabular}

